\title{
Mining Co-locations Under Uncertainty
}

\author{
Zhi Liu ${ }^{1}$, Yan Huang ${ }^{2}$ \\ ${ }^{1}$ zhiliu@my.unt.edu, ${ }^{2}$ huangyan@unt.edu \\ Computer Science and Engineering \\ University of North Texas
}

\begin{abstract}
A co-location pattern represents a subset of spatial features whose events tend to locate together in spatial proximity. The certain case of the co-location pattern has been investigated. However, location information of spatial features is often imprecise, aggregated, or error prone. Because of the continuity nature of space, over-counting is a major problem. In the uncertain case, the problem becomes more challenging. In this paper, we propose a probabilistic participation index to measure co-location patterns based on the well-known possible world model. To avoid the exponential cost of calculating participation index from all possible worlds, we prove a lemma that allows for instance centric counting, avoids over-counting, and produces the same results as using possible world based counting. We use this property to develop efficient mining algorithms. We observed through both algebraic analysis and extensive experiments that the feature tree based algorithm outperforms uncertain Apriori algorithm by an order of magnitude not only for co-locations of large sizes but also for datasets with high level of uncertainty. This is an important insight in mining uncertainty co-locations.
\end{abstract}

\section{Introduction}

A co-location represents a subset of spatial features whose events frequently appear together in spatial proximity. In Epidemiology, incidents of different but related diseases occur in different places. These diseases may exhibit co-location patterns where some types of diseases tend to occur in spatial proximity. In Ecology, different types of animals can be observed in different locations. There exist patterns such as symbiotic relationship and predator-prey relationship. Different types of crimes committed and different types of road accidents may also exhibit co-location. Many spatial data are uncertain with approximations and errors in real world. In Epidemiology, the occurrence of a disease may not be geo-located precisely and may be often associated with several locations, e.g. home and work place. In Ecology, observation of spices is often imprecise. Finding co-locations under uncertainty is useful for these domains.

In the problem of mining certain co-locations, a set $S$ of spatial features is given and each spatial feature $s$ is associated with a set of events $s . E$. The spatial feature of a given event $e$ is denoted as $s(e)$. A set of events $E$ is supporting a subset of spatial feature $S^{\prime} \subseteq S$ if: (1) $E$ forms a clique using a user given distance threshold; (2) for any $e_{1} \in E, e_{2} \in E$ and $e_{1} \neq e_{2}$, we 


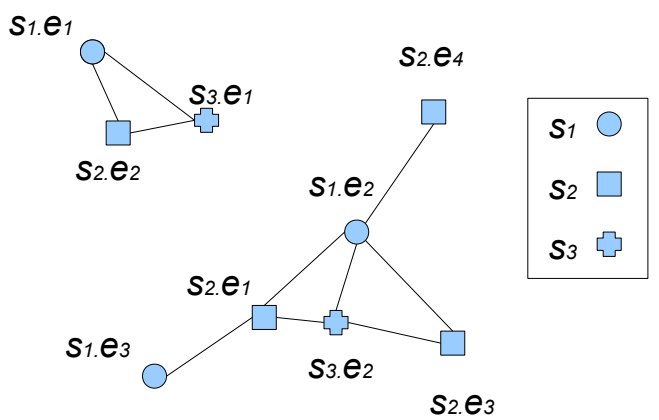

Fig. 1: Example Co-location. Three spatial features: $\left\{s_{1}, s_{2}, s_{3}\right\} . s_{1}$ has three events, $s_{2}$ has four events, and $s_{3}$ has two events.

have $s\left(e_{1}\right) \neq s\left(e_{2}\right) ;(3) \cup_{e \in E}\{s(e)\}=S^{\prime}$. The participation ratio $P R\left(s, S^{\prime}\right)$ of a spatial feature $s$ in a subset of spatial feature $S^{\prime} \subseteq S$ is the probability of an event of $s$ participating in a supporting clique of $S^{\prime}$. For example, in Figure 1, there are three spatial features $S=\left\{s_{1}, s_{2}, s_{3}\right\}$. Two events of different spatial features are connected if their distance is less than a user specified threshold. Feature $s_{2}$ has four events and three of them participate in a clique supporting $\left\{s_{1}, s_{2}, s_{3}\right\}$. So $P R\left(s_{2},\left\{s_{1}, s_{2}, s_{3}\right\}\right)$ is $3 / 4$. Then participation index $P I\left(S^{\prime}\right)$ is defined as $P I\left(S^{\prime}\right)=\min _{s \in S^{\prime}}\left\{P R\left(s, S^{\prime}\right)\right\}$. In Figure 1, $P I\left(s_{1}, s_{2}, s_{3}\right)=\min \{2 / 3,3 / 4,2 / 2\}=2 / 3$. The problem of co-location mining is to find all subsets of spatial features with participation indices above a user defined threshold.

When an event is uncertain, there are two main challenges: (1) How to define participation ratio and participation index in a probabilistic manner? (2) How to efficiently find co-locations when the number of uncertain events is large? Let us assume a simple dataset of 5 spatial features. Let each spatial feature have 4 events and each event has 3 possible locations. Then we will be $3^{4^{5}}=3.49 \times 10^{9}$ possible worlds.

\section{Contributions}

- We formulate a framework for mining uncertainty co-locations. The framework is based on possible world model. The participation index to measure co-location with uncertainty is defined in a probabilistic manner;

- We prove a lemma that allows an instance centric algorithm to be developed which is significantly faster than using all possible worlds to compute the participation index;

- We propose an Uncertain Apriori co-location mining algorithm (UApriori) and an Uncertain Feature Tree based algorithm (UFTree) to efficiently mine co-locations under uncertainty; we further propose event-based optimizations and table search techniques for optimizing these algorithms;

- Algebraic analysis and experiments on a large Shanghai taxi trajectory dataset and synthetic datasets show the effectiveness and efficiency of our 
proposed model and algorithms. We observed that the UFTree algorithm outperforms UApriori by an order of magnitude not only for co-locations of large sizes but also for datasets with high level of uncertainty. This is an important insight in mining uncertainty co-locations.

\section{Problem Definition}

We follow the commonly used uncertainty model Block-Independent Disjoint Scheme [3] to define the problem of uncertainty co-location. A probabilistic spatial feature $s$ is given by a set of uncertain events $s=\left\{e_{1}, e_{2}, \ldots\right\}$. An uncertain event $e_{i}$ is represented by a set of $d$-dimensional points $u_{1}, u_{2}, \ldots$ reflecting all possible instances of $e_{i}$. Each instance $u_{k}$ is assigned with a probability $P\left(u_{k}\right)$ denoting the probability that $e_{i}$ appears at $u_{k}$. Let $h$ be a user given distance threshold. In Figure 2(a), there are three spatial features. Spatial feature $s_{1}$ has three uncertain events, spatial feature $s_{2}$ has four uncertain events, and spatial feature $s_{3}$ has two uncertain events. Each instance of an uncertain event of $s_{1}$, $s_{2}$, and $s_{3}$ is represented by a circle, a square, and a plus sign respectively. Probabilities are assigned to instances of an uncertain event that sums up to 1 within the event. Two instances of different spatial features that are within a user given threshold $h$ are connected by a line. In this example, $s_{1}, s_{2}, s_{3}$ may be three animal species, e.g. $s_{1}=$ Egyptian plover, $s_{2}=$ Nile crocodile, and $s_{3}=$ monitor lizard. An event of $s_{1}$ represents a particular Egyptian plover, e.g. plover Smith. And the instance of the plover Smith is an location where Smith is spotted.

A possible world $w_{s}=u_{1}^{s}, u_{2}^{s}, \ldots$ of a spatial feature $s$ is a set of instances containing one instance from each event of $s$ and occurring with a probability of $P\left(w_{s}\right)=\prod_{k} P\left(u_{k}^{s}\right)$. Let $W_{s}$ be the set of all possible worlds for $s$, then $\sum_{w \in W_{s}} P(w)=1$. We are given a set $n$ of probabilistic spatial features $S=\left\{s_{1}, s_{2}, \ldots, s_{n}\right\}$, a possible world $w_{S}$ is given by the combination of a possible world of each spatial feature, i.e. $w_{s_{1}}, w_{s_{2}}, \ldots, w_{s_{n}}$ with a probability of $P\left(w_{S}\right)=\prod_{i} P\left(w_{s_{i}}\right)$. Obviously, let $W_{S}$ be the set of all possible world for $S$, then $\sum_{w \in W_{S}} P(w)=1$ as well.

Definition[Complete Possible World] Given a subset of probabilistic spatial features $S, W_{S}^{c}$ includes all the instances of all the events of $S$ and is called the complete possible world with respect to $S$. For any instance, we use $e(u)$ to denote the event associated with $u$ and use $s(u)$ to denote the spatial feature of $u$.

All of the instances in Figure 2(a) is an example of complete possible world of $\left\{s_{1}, s_{2}, s_{3}\right\}$.

Definition[Supporting Clique] A set of instances $c$ in the complete world $w_{S^{\prime}}^{c}$ is supporting a subset of spatial feature $S^{\prime} \subseteq S$ if: (1) $c$ forms a clique using a user given distance threshold; $(2) s\left(u_{1}\right) \neq s\left(u_{2}\right)$ (which also implies $\left.e\left(u_{1}\right) \neq e\left(u_{2}\right)\right)$ for any $u_{1} \in c, u_{2} \in c$ and $u_{1} \neq u_{2} ;(3) \cup_{u \in U}\{s(u)\}=S^{\prime} . c$ is called a supporting clique of $S^{\prime}$.

In Figure 2(a), $\left\{s_{1}, s_{2}, s_{3}\right\}$ has 6 supporting cliques. However, not all of them can appear together in the same possible worlds as explained later. 


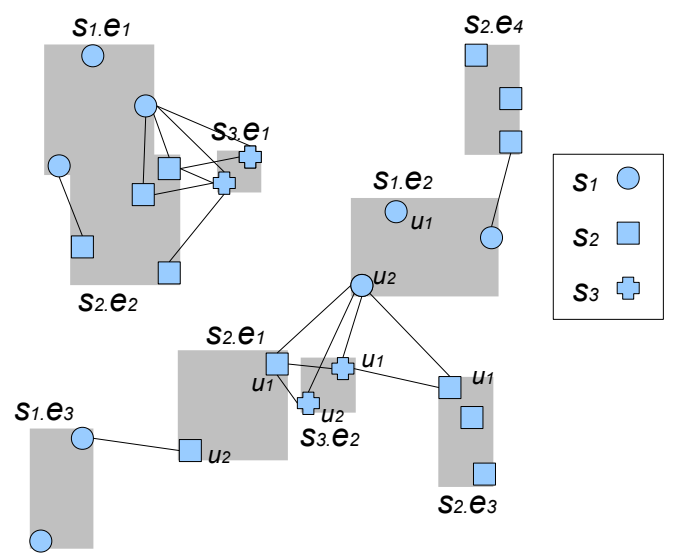

(a) Example of Uncertainty Co-location

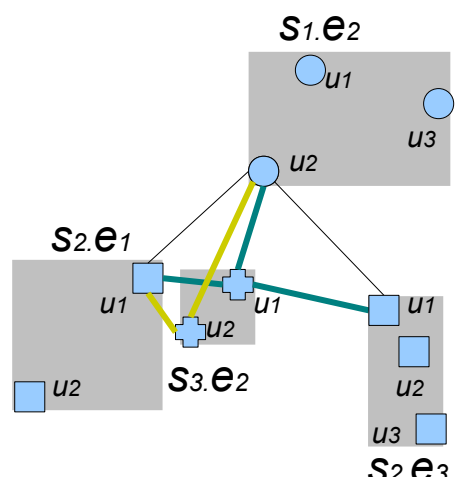

(b) Instance Centric Counting

Fig. 2: In 2(a), feature $s_{1}$ has three uncertain events, i.e. $\left\{s_{1} \cdot e_{1}, s_{1} \cdot e_{2}, s_{1} \cdot e_{3}\right\}$. Event $s_{1} . e_{1}$ has three uncertain instances.

The participation ratio and index are used to prune the co-locations with low prevalence. Different from certain cases, the participation ratio can not be directly calculated by counting the instances participating in a supporting clique. In an uncertain case, the participation ratio of an instance will be determined by both the probability of possible worlds and the supporting cliques it participates.

Definition[Participation Ratio of an Instance] For a subset $S^{\prime}$ of $S$ and a user given distance threshold, the participation ratio of an instance $u$ of spatial feature $s_{i}$ in $S^{\prime}$ is defined as the sum of probabilities of all the possible worlds that instance $s_{i} . u$ participates in a supporting clique of $S^{\prime}$ and this can be calculated as:

$$
P R\left(u, S^{\prime}\right)=\sum_{\left\{w \mid \exists c, u \in c, c \subseteq w, w \in W_{S^{\prime}}, c \text { supports } S^{\prime}\right\}} p(w)
$$

In Figure 2(b), $P R\left(s_{1} \cdot e_{2} \cdot u_{2},\left\{s_{1}, s_{2}, s_{3}\right\}\right)$ is the sum of the probabilities of all the possible worlds that contain at least one supporting clique of $\left\{s_{1}, s_{2}, s_{3}\right\}$ with $s_{1} \cdot e_{2} \cdot u_{2}$ participating. In this example, it includes all of the worlds that contain one or more of the three supporting cliques that $s_{1} \cdot e_{2} \cdot u_{2}$ participates in the figure. Since $s_{1} . e_{2}, s_{2} . e_{1}, s_{3} . e_{2}$ and $s_{2} . e_{3}$ have $3,2,2,3$ possible instances respectively, the total number of possible world should be 36 . Among those, 3 possible worlds have a supporting clique of $\left\{s_{1} \cdot e_{2} \cdot u_{2}, s_{2} \cdot e_{1} \cdot u_{1}, s_{3} \cdot e_{2} \cdot u_{1}\right\}, 3$ contain that of $\left\{s_{1} \cdot e_{2} \cdot u_{2}, s_{2} \cdot e_{1} \cdot u_{1}, s_{3} \cdot e_{2} \cdot u_{2}\right\}$, and 2 contain that of $\left\{s_{1} \cdot e_{2} \cdot u_{2}, s_{2} \cdot e_{3} \cdot u_{1}, s_{3} \cdot e_{2} \cdot u_{1}\right\}$. Given equal probability of instances, all of the possible worlds have the same probability of $1 / 36$. However, one possible world contains both of the cliques $\left\{s_{1} \cdot e_{2} \cdot u_{2}, s_{2} \cdot e_{1} \cdot u_{1}, s_{3} \cdot e_{2} \cdot u_{1}\right\}$ and $\left\{s_{1} \cdot e_{2} \cdot u_{2}, s_{2} \cdot e_{3} \cdot u_{1}, s_{3} \cdot e_{2} \cdot u_{1}\right\}$. So there are total- 
ly $3+3+2-1=7$ possible worlds that $s_{1} \cdot e_{2} \cdot u_{2}$ participates in a supporting clique of $\left\{s_{1}, s_{2}, s_{3}\right\}$. The participation ratio of $s_{1} \cdot e_{2} \cdot u_{2}$ should be $P R\left(s_{1} \cdot e_{2} \cdot u_{2}\right)=$ $7 \times(1 / 36)$.

Definition[Probabilistic Participation Ratio] Let $s_{i} . U$ be all possible instances of $s_{i}$ where $s_{i} \in S^{\prime}$ and $\left|s_{i}\right|$ be the number of events of $s_{i}$. The probabilistic participation ratio (short as participation ratio or PR here after) of $s_{i}$ is:

$$
P R\left(s_{i}, S^{\prime}\right)=\frac{1}{\left|s_{i}\right|} \sum_{u \in s_{i} . U} P R\left(u, S^{\prime}\right)
$$

Definition[Probabilistic Participation Index] For a subset $S^{\prime} \subseteq S$, the probabilistic participation index (short as participation index or PI) $P I\left(S^{\prime}\right)$ of $S^{\prime}$ is defined as follows:

$$
P I\left(S^{\prime}\right)=\min P R\left(s_{i}, S^{\prime}\right)
$$

The problem of mining co-location under uncertainty is to find all subsets of spatial features with participation indexes above a user given threshold $\theta$.

\section{Instance Centric Counting}

The naive way to calculate the participation ratio of an instance in $S^{\prime}$ is to enumerate all the possible worlds and then sum up the probabilities of the worlds where the instance participates in a supporting clique of $S^{\prime}$. However this method is very expensive as the number of possible worlds is very large. We propose an instance centric calculation of the participation ratio, taking supporting cliques involved into consideration. However, in this method, avoiding over-counting is a challenge. We prove a Lemma to allow instance centric counting which will enable efficient algorithms to be developed. Using this method, we can get the same result as summing up possible worlds above. We first define the relationship between a possible world and a clique.

Definition[Clique Probability] For a supporting clique $c$ of $S^{\prime}$, the probability $P(c)$ is the sum of the probability of all of the worlds which contains the clique $c$. And it can be represented as:

$$
P\left(c, S^{\prime}\right)=\sum_{\left\{w \mid w \in W_{S^{\prime}}, c \in w\right\}} P(w)
$$

Finding all the possible worlds that contain $c$ is very expensive. However, it is easy to prove the lemma 1 . The proof is similar of that of lemma 2 and is omitted due to space constraint.

Lemma 1 For a supporting clique $c$ of $S^{\prime}$, the probability $P\left(c, S^{\prime}\right)$ is equivalent to:

$$
P\left(c, S^{\prime}\right)=\prod_{u \in c} P(u)
$$


If an instance $u$ only participates in one supporting clique, then the participation ratio of $u$ is the probability of the clique. However, an instance can participate in multiple cliques in the same world, resulting in over counting if we simply sum up the probabilities of all the supporting cliques that $u$ participates (naive PR calculation). For example, Figure 2(b) is a subset of Figure 2(a) which includes all events and cliques that relate to $s_{1} \cdot e_{2} \cdot u_{2}$. The two instances of $s_{3} \cdot e_{2}$ cannot happen at the same time (yellow lines and green lines could not happen in the same world). So not all three cliques can happen in the same world. However, clique $\left\{s_{1} \cdot e_{2} \cdot u_{2}, s_{2} \cdot e_{1} \cdot u_{1}, s_{3} \cdot e_{2} \cdot u_{1}\right\}$ and $\left\{s_{1} \cdot e_{2} \cdot u_{2}, s_{2} \cdot e_{3} \cdot u_{1}, s_{3} \cdot e_{2} \cdot u_{1}\right\}$ can co-exist, resulting in over counting of the worlds that contain both if we use the naive PR calculation.

Definition[Coexistence] Two supporting cliques $c_{1}$ and $c_{2}$ of $S^{\prime}$ can coexist, denoted as $\odot\left(c_{1}, c_{2}\right)=1$, in the same possible world under the following condition: $\forall u_{1} \in c_{1}, u_{2} \in c_{2}$, if $e\left(u_{1}\right)=e\left(u_{2}\right)$, then $u_{1}=u_{2}$, where $e(u)$ denotes the event associated with the instance $u$.

Definition[Coexistence of a Set of Supporting Cliques] A set of supporting cliques $C$ can coexist and is denoted as $\odot(C)=1$ if every pair of the cliques can coexist.

Definition[Probability of Coexisting Clique Set] The probability of a set of coexisting supporting cliques $C$ of $S^{\prime}$ is the sum of the probabilities of those possible worlds in which $C$ happens:

$$
P\left(C, S^{\prime}\right)=\sum_{\left\{w \mid \forall c \in C, c \subseteq w, w \in W_{S^{\prime}}\right\}} P(w)
$$

Lemma 2 The probability of a set of coexisting supporting cliques $C$ of $S^{\prime}$ can be calculated as:

$$
P\left(C, S^{\prime}\right)=\prod_{u \in I} P(u)
$$

where $I$ is the union of all instances of the events of the cliques in $C$.

Proof: Let $E$ be the set of all events. For any $E^{\prime} \subseteq E$, it is easy to see that the sum of the probabilities of all possible worlds $W_{E^{\prime}}$ that include an instance from each event in $E^{\prime}$ is 1 . The probability of coexisting clique set is the sum of all possible worlds which contain the coexisting supporting clique set $C$. We can divide $E$ into two parts: $E_{1}$ is the set of events having an instance in the coexisting cliques and $E_{2}$ is the complementary set. So a possible world that contains $C$ can also contain any other possible worlds of $E_{2}$. So the summation can be calculated as $\prod_{u \in I} P(u) \times\left(\sum_{w \in W_{E_{2}}} P(w)\right)$. We know that $\sum_{w \in W_{E_{2}}} P(w)=1$, so the probability of a set of coexisting supporting cliques is $\prod_{u \in I} P(u)$.

From lemma 1 we know that the probability of a clique $c_{1}$ is the sum of all of the possible worlds that contain the clique. However, those possible worlds can contain both cliques $c_{1}$ and $c_{2}$. So the probabilities of the possible worlds which contain both cliques will be counted two times if they can coexist when counting the participation ratio of an instance. To summarize, when there are 
coexisting cliques and they contribute to the participation of the same instance, over counting will happen. We define such cliques as star supporting cliques.

Definition[Star Supporting Clique Set] All the supporting cliques of $S^{\prime}$ that an instance $u$ participates is called the star supporting clique set of $u$ in $S^{\prime}$ and is denoted as $\star\left(u, S^{\prime}\right)$.

Lemma 3 To an instance $s_{i} . u_{k}$ in a complete possible world, the star supporting clique set $\star\left(s_{i} \cdot u_{k}, S^{\prime}\right)$ of $S^{\prime}$ can be used to calculate the participation ratio of the instance as follows where $l=\left|\star\left(s_{i} . u_{k}, S^{\prime}\right)\right|$ :

$$
\begin{aligned}
& P R\left(s_{i} . u_{k}, S^{\prime}\right)=\sum_{c_{j} \in \star\left(s_{i} . u_{k}, S^{\prime}\right)} P\left(c_{j}, S^{\prime}\right) \\
& -\sum_{c_{1} \in \star\left(s_{i} . u_{k}, S^{\prime}\right), c_{2} \in \star\left(s_{i} . u_{k}, S^{\prime}\right), \odot\left(c_{1}, c_{2}\right)} P\left(c_{1} \cup c_{2}, S^{\prime}\right)+\ldots \\
& +(-1)^{l+1} \sum_{c_{1} \in \star\left(s_{i} . u_{k}, S^{\prime}\right), . . c_{l} \in \star\left(s_{i} . u_{k}, S^{\prime}\right), \odot\left(c_{1}, . . c_{l}\right)} P\left(c_{1} . . \cup c_{l}, S^{\prime}\right)
\end{aligned}
$$

Proof: From the definition of participation ratio, $P R\left(u_{k}\right)=\sum_{w_{i} \in W_{S^{\prime}}} P\left(w_{i}\right)$, where $W_{S^{\prime}}$ is the set of possible worlds which instance $u_{k}$ participates in forming a supporting cliques of $S^{\prime}$. So we can prove lemma 3 by checking whether the probability of each possible world in $W_{S^{\prime}}$ has been counted and only counted once. For each $w_{i}$ in $W_{S^{\prime}}$, let us assume that $w_{i}$ has $n$ coexisting cliques $\left\{c_{1}, c_{2} \ldots c_{n}\right\}$. From the definition of the probability of coexisting clique set, we know that every time we calculate $P\left(C, S^{\prime}\right)$, we count the probability of $w_{i}$ once. Here $C$ represents any possible combinations of coexisting cliques in $w_{i}$. So when we calculate $P\left(c_{1}\right)$ as well as $P\left(c_{1} \cup c_{2}\right)$ and any other combinations, we count $P\left(w_{i}\right)$ once. In lemma 3 , for $w_{i}$, each of $P\left(c_{1}\right) \ldots P\left(c_{n}\right)$ is added to the probability of $w_{i}$ once. Each of $P\left(c_{1} \cup c_{2}\right) \ldots P\left(c_{n-1} \cup c_{n}\right)$ subtracts $P\left(w_{i}\right)$ once. So $P\left(w_{i}\right)$ has been counted $C_{n}^{1}-C_{n}^{2}+\ldots(-1)^{n+1} C_{n}^{n}=1$ times.

Lemma 4 (Anti-monotone Property) The participation index is anti-monotone with respect to the number of features in the co-location.

Proof: Let $l, l^{\prime}$ be two co-locations and $l^{\prime} \subseteq l$. We use $W_{l}$ and $W_{l^{\prime}}$ to represent the set of possible worlds which have supporting cliques of these two co-locations. For each possible world $w$ in $W_{l}, w$ contains supporting cliques of $l$. Because $l^{\prime} \subseteq$ $l, w$ will also has supporting cliques of $l^{\prime}$. So if $w \in W_{l}$, then $w_{i} \in W_{l^{\prime}}$. Therefore, $W_{l} \subseteq W_{l^{\prime}}$. From the definition of participation index, $P I(l)=\sum_{w \in W_{l}} P(w)$ and $P I\left(l^{\prime}\right)=\sum_{w \in W_{l^{\prime}}} P(w)$, we can conclude that $P I\left(l^{\prime}\right) \geq P I(l)$.

\section{Mining Co-location from Uncertain Data}

In this section, we describe the framework of mining uncertain co-locations. While participation ratio can be calculated by counting supporting cliques [10] 
in the certain case, the participation ratio of uncertain co-locations is calculated by the probabilities of instances. Thus we divide the process of mining co-location in two steps, (1) finding uncertainty co-locations and their supporting cliques; (2) calculating the participation ratios. In section 4.1, we propose an Apriori based algorithm to generate supporting cliques under uncertainty. Section 4.2 will present the feature tree driven method combined with maximal clique method. We propose event-based pruning and clique-feature table searching to reduce the computational cost. In section 4.3, we will present the algorithm for calculating participation ratios and section 4.4 presents algebraic analysis of the computational complexity.

In the following part, we use $C_{k}$ to represent a set of size $k$ cliques, use $L_{k}$ to represent a set of size $k$ co-locations and use $C_{M}$ to represent a set of maximal cliques.

\subsection{Uncertain Apriori (UApriori) Co-location Miner}

We first propose an Apriori like algorithm in the instance level and will present event level pruning in section 4.1. We process in the uncertain instance level to generate the supporting cliques for each co-location. Algorithm 1 shows the process of how to generate uncertainty co-location by Apriori algorithm. Here we use an example to illustrate. Figure 3(a) is the Apriori-gen process for the example in figure 2(b). We use plane sweep algorithm to get the neighbor relation of between the instances of $S_{1}, S_{2}, S_{1}, S_{3}$ and $S_{2}, S_{3}$ (the first three tables). They are also the supporting cliques $C_{2}$ of size-2 co-locations $L_{2}$. Assuming all of them have a participation index greater than the threshold, we can generate size- 3 co-locations by them. First we generate the supporting cliques for the co-location $\left\{s_{1}, s_{2}, s_{3}\right\}$ from $C_{2}$ through joins. For example, we use the size-2 cliques $\left\{s_{1} \cdot e_{2} \cdot u_{2}, s_{2} \cdot e_{1} \cdot u_{1}\right\}$ and $\left\{s_{1} \cdot e_{2} \cdot u_{2}, s_{3} \cdot e_{2} \cdot u_{1}\right\}$ in $C_{2}$ to generate the size-3 supporting clique $\left\{s_{1} \cdot e_{2} \cdot u_{2}, s_{2} \cdot e_{1} \cdot u_{1}, s_{3} . e_{2} . u_{1}\right\}$. When generating the new supporting clique, we calculate the distance between $s_{2} \cdot e_{1} \cdot u_{1}$ and $s_{3} \cdot e_{2} \cdot u_{1}$ by their coordinates instead of searching the $c_{2}$ table to find the record of clique $\left\{s_{2} \cdot e_{1} \cdot u_{1}, s_{3} \cdot e_{2} \cdot u_{1}\right\}$.

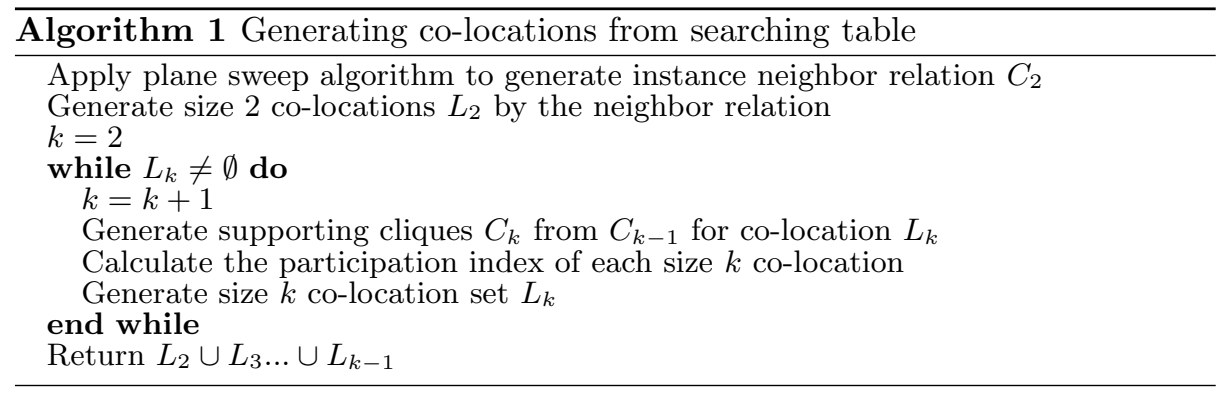




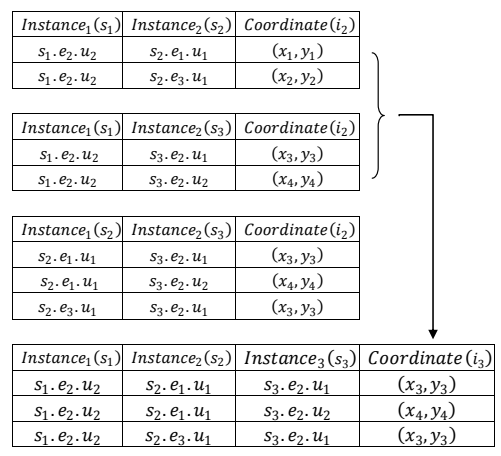

(a) Process of Apriori-gen

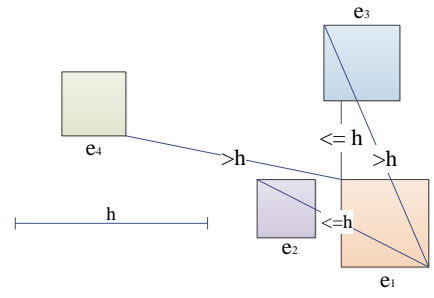

(b) Event Level Pruning

Fig. 3: Example for the process of UApriori

Event Level Pruning (UApriori-E) However in uncertainty, each event of a feature may have many uncertain instances. Both algebraic and experiments show that with large number of instances, the join process to generate cliques become inefficient. To optimize this process, we propose an event level pruning approach to apply event neighbor relation checking first and only proceed to instance level join when the minimal distance between two events are within user given distance.

There are three relations between two events: the minimum distance larger than threshold $h$, the maximal distance less than $h$, and Distance $_{\text {Max }}>h \cap$ Distance $_{\text {Min }}<h$. For the first case, such as $e_{1}$ and $e_{4}$ in figure 3(b), we can prune $e_{4}$ directly. For the second, such as $e_{1}$ and $e_{2}$, all instances in $e_{2}$ can form neighbor relation with instances in $e_{1}$ without calculating the distances between them. We only need to calculate the distance between instances in the third case.

\subsection{Uncertain Feature Tree Co-location Miner (UFTree)}

Feature tree has been used in mining long pattern association rules. However, the main challenge in using the same structure in co-location mining is the lack of given set of transactions. In this paper, we propose feature tree based methods in mining uncertain co-locations that deal with the lack of transactions. In the first step, we use the plane sweep algorithm and the Bron-Kerbosch algorithm [4] to find all maximal cliques in the complete possible world (optimization will be discussed shortly) and add them into $C_{M}$. A naive way to generate the supporting cliques is to enumerate all sub-cliques of every maximal clique and map them into different co-locations. But without a pruning process, the cost is prohibitive especially when some large size cliques exist. Here we build a searching table to help generating the supporting cliques and the co-location set. Algorithm 2 describes the framework of this process. 


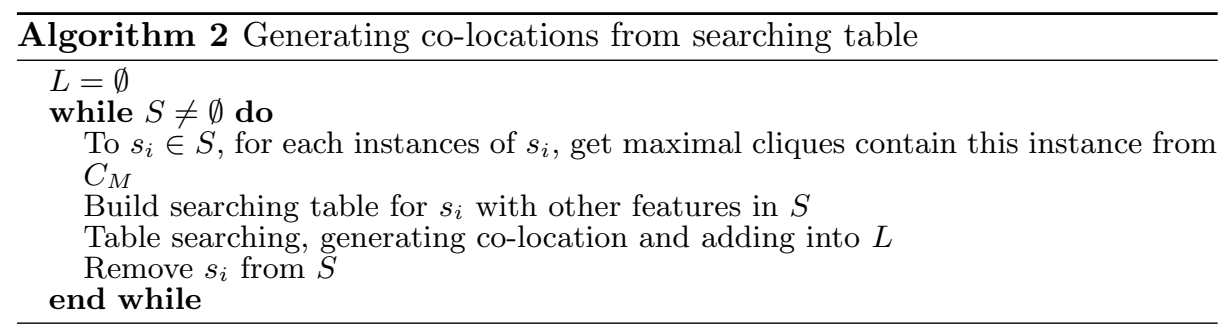

Figure 4 is an example for building searching table. $\left\{c_{1}, c_{2}, . . c_{6}\right\}$ is the set of maximal cliques $C_{M}$ and $\left\{s_{1}, s_{2}, . . s_{6}\right\}$ is the set of features $S$. For $s_{1}$ in $S$, we first get the maximal cliques containing instances of $s_{1}:\left\{c_{1}, c_{2}, c_{3}, c_{4}, c_{5}\right\}$. Then those maximal cliques will be mapped into the searching table in figure 4 . After the co-location generating step, $s_{1}$ will be removed from $S$ and will not appear in the searching table built after this. For example, when we build the searching table for $s_{2}$, instances from $s_{1}$ will not be included in the table.

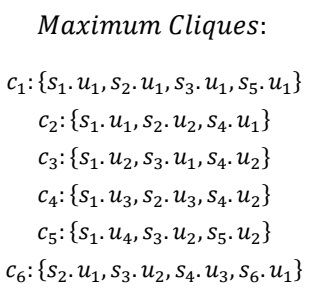

Searching Table
\begin{tabular}{|c|c|c|c|c|c|}
\hline & $c_{1}$ & $c_{2}$ & $c_{3}$ & $c_{4}$ & $c_{5}$ \\
\hline$s_{1}$ & $s_{1} \cdot u_{1}$ & $s_{1} \cdot u_{1}$ & $s_{1} \cdot u_{2}$ & $s_{1} \cdot u_{3}$ & $s_{1} \cdot u_{4}$ \\
\hline$s_{2}$ & $s_{2} \cdot u_{1}$ & $s_{2} \cdot u_{2}$ & & $s_{2} \cdot u_{3}$ & \\
\hline$s_{3}$ & $s_{3} \cdot u_{1}$ & & $s_{3} \cdot u_{1}$ & & $s_{3} \cdot u_{2}$ \\
\hline$s_{4}$ & & $s_{4} \cdot u_{1}$ & $s_{4} \cdot u_{2}$ & $s_{4} \cdot u_{2}$ & \\
\hline$s_{5}$ & $s_{5} \cdot u_{1}$ & & & & $s_{5} \cdot u_{2}$ \\
\hline
\end{tabular}

Fig. 4: Searching Table

To search this table and generate co-locations, we introduce the searching strategy on a tree-based structure. The breadth first searching with follow set pruning approach.

Uncertainty Feature Tree Searching with Follow Set Pruning (UFTreeFP algorithm) We introduce the follow set searching strategy to generate co-locations on the table. We first present key definitions for this strategy.

Definition[Pre-Node Set] Node $\mathcal{N}$ is a node in the feature tree. The prenode set $\operatorname{Pre}\{\mathcal{N}\}$ of $\mathcal{N}$ is the set of nodes which have the same father node as $\mathcal{N}$ and at the left side of $\mathcal{N}$ in the feature tree.

Definition[Follow set] The follow set of node $\mathcal{N}$ is a set of features which may form co-locations with the features in node $\mathcal{N}$.

For example, for the root node of the tree in figure $5(\mathrm{a})$, the set of $\left\{s_{2}, s_{3}, s_{4}, s_{5}\right\}$ is the follow set of this root node. Feature $s_{1}$ will be combined with each of them to generate new co-locations in the next level of this tree. 


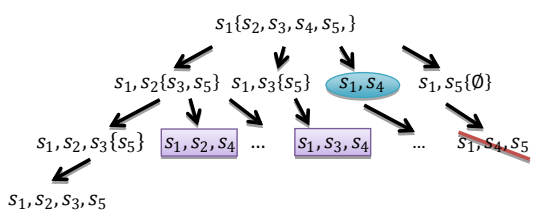

(a) Searching tree

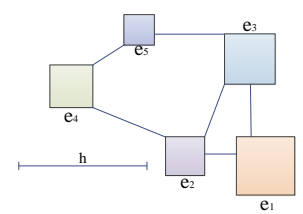

(b) Finding maximal cliques at event level

Fig. 5: Strategie on searching the table

Algorithm 3 describes the process of breadth-first search to generate colocations related to $s_{i}$. When searching the table, we will generate the follow set for each node. First we set the follow set of the root node as $S_{s_{i}}-s_{i}$ and add the root node to the node list. Then we process each node in the node list. For every node, we combine the feature set of the node with each feature $S_{F i}$ in the follow set and get a new co-location. If the participation index of the new co-location is greater than the threshold, this node will be added into the node list and the feature $S_{F i}$ will be added into the follow sets of every pre-node of this node.

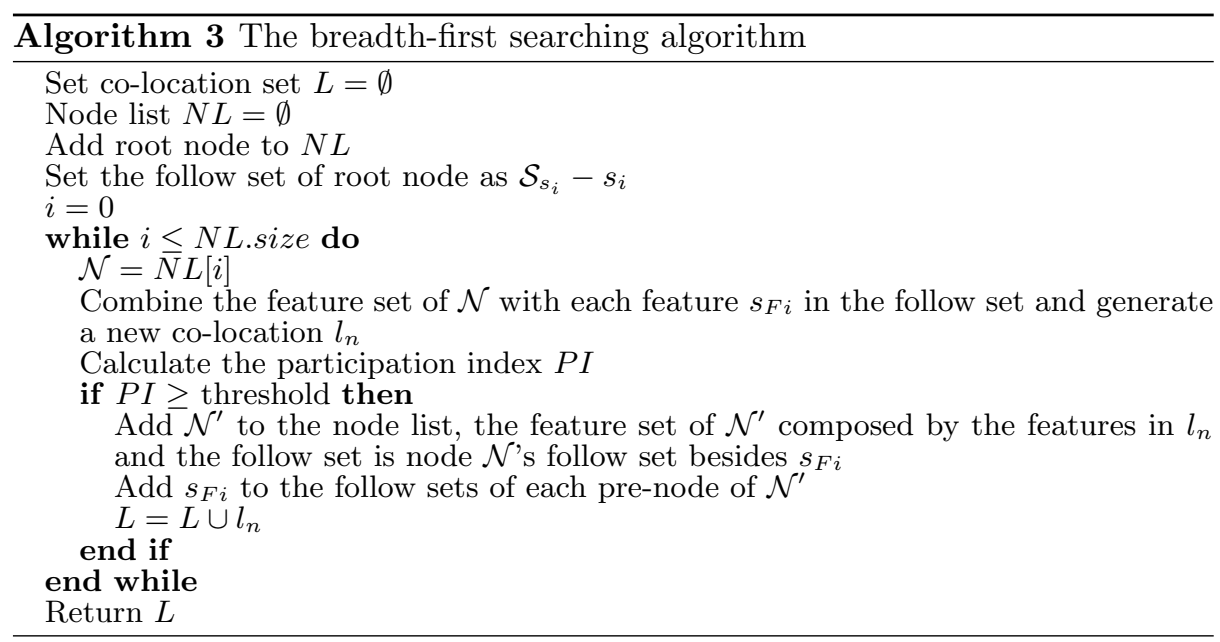

Figure 5(a) is an example for breadth-first search. During the search for the node of $\left\{s_{1}, s_{3}\right\}$, if the participation ratio is greater than the threshold, $s_{3}$ will be added into the follow set of node $\left\{s_{1}, s_{2}\right\}$. The participation ratio of $\left\{s_{1}, s_{4}\right\}$ is less than the threshold, so the sub-tree of this node $\left\{s_{1}, s_{4}, s_{5}\right\}$ will be pruned and $s_{4}$ will not be added into the follow sets of the pre-nodes of $\left\{s_{1}, s_{4}\right\}$. So the node of $\left\{s_{1}, s_{2}, s_{4}\right\}$ and $\left\{s_{1}, s_{3}, s_{4}\right\}$ will also be pruned. 
The process of finding maximal cliques can be computational expensive with high density of instances. To avoid this, we generated maximal cliques in the event level at first and used this as an index to generate instance level cliques used in the searching table. For example, in the figure $5(\mathrm{~b})$, there are four maximal event cliques: $\left\{e_{1}, e_{2}, e_{3}\right\},\left\{e_{2}, e_{4}\right\},\left\{e_{3}, e_{5}\right\}$ and $\left\{e_{4}, e_{5}\right\}$.

\subsection{Calculating the Participation Index}

We have proposed lemma 3 to calculate the participation ratio of each possible instance. If there was no coexisting cliques, lemma 3 can be represented as: $P R\left(s_{i} \cdot u_{k}, S^{\prime}\right)=\sum_{c_{j} \in \star\left(s_{i} . u_{k}, S^{\prime}\right)} P\left(c_{j}, S^{\prime}\right)$. So we can calculate all the participation ratios of possible instances by calculating the probability of each supporting clique and add the probability to the ratio of the instances belonging to this clique. If $c_{i}$ and $c_{j}$ are two coexisting cliques and $\left\{u_{1}, u_{2} \ldots u_{n}\right\}$ are their common possible instances, to these instances, their participation ratios $P R(u)$ will be deducted by $P\left(c_{i} \cup c_{j}\right)$ according to the lemma 3 . So we need to calculate the probabilities of different combinations of coexisting cliques and add or subtract the results to the ratios of instances shared by those cliques according to lemma 3. After getting all of the participation ratios, we can calculate the participation indexes of co-locations by the method described in the definition of probabilistic participation ratio and participation index.

\subsection{Computational Complexity}

Table 1 summarizes the parameters used in complexity analysis as well as in the experiment section. In the UApriori algorithm, when generating size $(k+1)$ co-locations from size $k$, the worst case is: for each supporting clique, we have to see if they have $k-1$ instances in common with other cliques. If we define $N_{C(k)}$ as the number of supporting cliques of size $\mathrm{k}$ co-locations, the cost of generating supporting cliques is: $O\left(\sum_{k=2}^{w-1}(k-1)\left|N_{C(k)}\right|\right)$. For the uncertain feature tree algorithm, the cost of generating step can be divided into three parts: finding out all maximal cliques, building searching table, and generating supporting cliques. Finding maximal cliques is a NP problem [4]. Thus we propose to do this step on event level in our approach to avoid high computational cost. For each feature, we use $N_{C_{L}}$ as the average number of supporting cliques of each co-location and $N_{F_{i}}$ as the number of cliques related to each feature. So the cost of building the searching table is $|F| N_{F_{i}} N_{C_{L}}$. To generate supporting cliques for each colocation set, we need to search a line of the searching table and the worst case is $O\left(N_{F_{i}}\right)$. So the cost of UFTree algorithm is $O\left(|F| N_{F_{i}} N_{C_{L}}+N_{F_{i}} \sum_{k=2}^{w} \mid\right.$ $C(k) \mid)$.

\section{Experiments}

In this section, we will first compare the algorithms on different synthetic dataset$\mathrm{s}$ to show the influence of parameters. Since there were no algorithms solving 
Table 1: Parameters

\begin{tabular}{|c|c|}
\hline$N_{s}$ & Number of Features \\
\hline$O_{L}$ & The overlapping ratio of co-locations \\
\hline $\bar{I}$ & Average number of instances of an event \\
\hline$N_{L}$ & Number of co-locations \\
\hline $\bar{L}$ & Average length of co-locations \\
\hline $\bar{E}$ & Average number of events of each feature \\
\hline $\bar{C}$ & Average number of supporting cliques of a co-location in event level \\
\hline
\end{tabular}

the same problem with us, here we only compared our own method and analysis the results. Then we will use the Shanghai taxi trip dataset to illustrate the application of our algorithms in real world.

\subsection{Synthetic Data Generation}

Our model of the synthetic data is similar of that of paper [10]. Parameters in table 1 are used to generate synthetic data. We generated uncertainty datasets with a broad range of values for the chosen parameters to evaluate the performance of those algorithms. The length of co-location, the average number of supporting cliques, and the average number of instances in an event will be picked from three Poisson distributions $P(\bar{L}), P(\bar{C})$ and $P(\bar{I})$. The generating step will be divided into three steps: 1 . Generating a co-location; 2. Generating event level supporting cliques for this co-location; 3. Transforming each event into possible instances using a Poisson distribution in an area of given size. We generated the datasets by setting different values for $\bar{L}$ (from 2 to 11), $\bar{E}$ (from 3 to 16 ), $\bar{I}$ (from 2 to 8 ), and setting 5 values for $O_{L}: 0,20 \%, 40 \%, 60 \%, 80 \%$.

\subsection{Performance on Synthetic Data}

Figure 6 presents the running time of the algorithms under different values of those parameters. We first compare those algorithms by changing the value of $\bar{E}$ and $\bar{I}$. The results are shown in figure 6(a) and 6(b). Figure 6(a) shows the influence of $\bar{E} . \bar{E}$ has little influence on the UFTree algorithm. However, with the increasing value of $\bar{E}$, the advantage of event pruning process becomes significant between UApriori and UApriori-E algorithms. In figure 6(b), we can see that with the increase of the average number of instance, the running times of both UApriori-E and UApriori algorithm increase quickly. But the UFTree algorithm still use a very short time. This phenomenon illustrates that generating cliques in the searching table performs much better than the Apriori-gen process. From figure 6(c), we can conclude that the overlapping ratio has litter influence on those algorithms. In figure $6(\mathrm{~d})$, we tested the influence of the threshold of participation ratio on running time.

The UFTree algorithm performs much better due to its fast matching process and searching strategy. To illustrate this phenomenon, we can simplify the 
expression of the computational complexity and just concentrate on the generating process to analyze the computational cost. For UApriori, UApriori-E and UFTree algorithms, the worst case of generating the supporting cliques can be represented by $O\left((k-1)+(\bar{E} \times \bar{I})^{2}\right), O\left((k-1)+\bar{E}^{2}+\bar{E} \times(\bar{I})^{2}\right)$ and $O\left((\bar{E} \times \bar{I})^{2}\right)$ in generating the supporting cliques of a size $k$ co-locations. But with different values of parameters, the costs of UApriori and UFTree algorithm are typically far less than that. For example, if event from one feature can only form one event pair with another event and there is only one instance pair between them, the cost of UApriori and UFTree will become $O\left((k-1)+\bar{E}^{2}+\bar{E} \times \bar{I}^{2}\right)$ and $O(\bar{E})$. So with the increase of $\bar{L}$, the UApriori Algorithm becomes much more slower and the UFTree algorithms provides a much better performance. We can see that in the results of figure $6(\mathrm{e})$.

Tree based algorithm has been used in long pattern association rule mining. A significant advantage of this algorithm is its subset pruning strategy. For example, when we get $\left\{S_{1}, S_{2}, S_{3}\right\}$, we need not to test $\left\{S_{2}, S_{3}\right\}$ due to the antimonotone property. In our experiment mentioned above (figure 6(a) to 6(e)), we didn't use this strategy. In figure 6(f), we compared the UFTree algorithm with and without the subset pruning: UFTree-Subset Pruning and UFTree algorithm. The results show that subset pruning can improve the performance especially in mining co-locations of large sizes. The reason is larger sized co-locations contain more subsets (equal to the number of power set). From the results, we can see that when the size of co-locations is larger than 8, the UTFree-Subset Pruning method made a significant improvement.

\subsection{Experiments on Real Data}

Data for the framework is based on trips of 17,000 Shanghai taxis for one day (May 29, 2009); the dataset contains 432,327 trips. Each trip includes the starting and destination coordinates and the start/end time. Our real data experiment is based on the premise that if people from two or more areas often take taxi to some other common areas, these two or more areas may have some potential characteristics in common. For example, they may be all university areas or residential areas. Of course, people from adjacent areas also tend to have the same destinations because adjacent areas tend to have similar characteristics. But the taxi trips start and end coordinates can be different even when they have the same start and destination areas. For example, a university may have several entrances and people can get on or off taxis at any entrance. So it becomes an uncertainty co-location problem. The university area is an event and each entrance can be a possible instance in our uncertainty co-location model.

We generate the dataset according to our uncertainty model from the real world taxi trips. Firstly, we divided the region of Shanghai into $500 \times 300 \mathrm{~m}$ rectangles. Top 680 areas with more than 300 trip starting points are chosen as feature areas. Each area is a spatial feature. For each feature $s$, an area $e$ with a trip starting in $s$ and ending in $e$ is an event of $s$. The trip ending points in an event $e$ of $s$ is then clustered into small groups, each representing an instance 

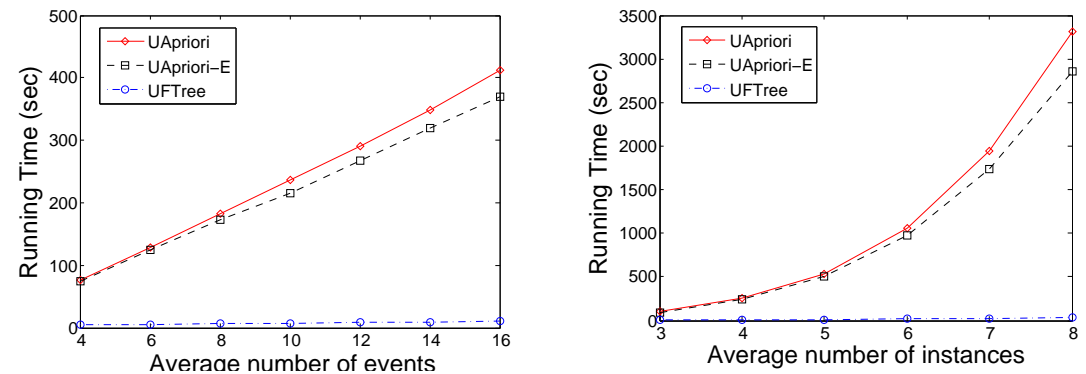

(a) $N_{L}=10 k, \bar{L}=4, \bar{E}: 4 \sim 16, \bar{I}=4$

(b) $N_{L}=20 k, \bar{L}=4, \bar{E}=5, \bar{I}: 3 \sim 8$
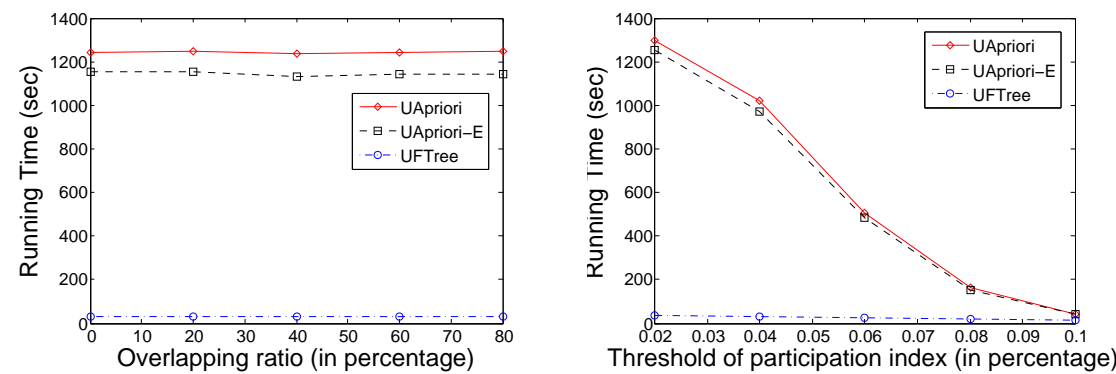

(c) $N_{L}=20 k, \bar{L}=5, \bar{E}=3, \bar{I}=3$, over- (d) $N_{L}=20 k, \bar{L}=6, \bar{E}=3, \bar{I}=3$,PI lapping $\operatorname{ratio}(\%): 0 \sim 80$ threshold $(\%): 0.02 \sim 0.1$
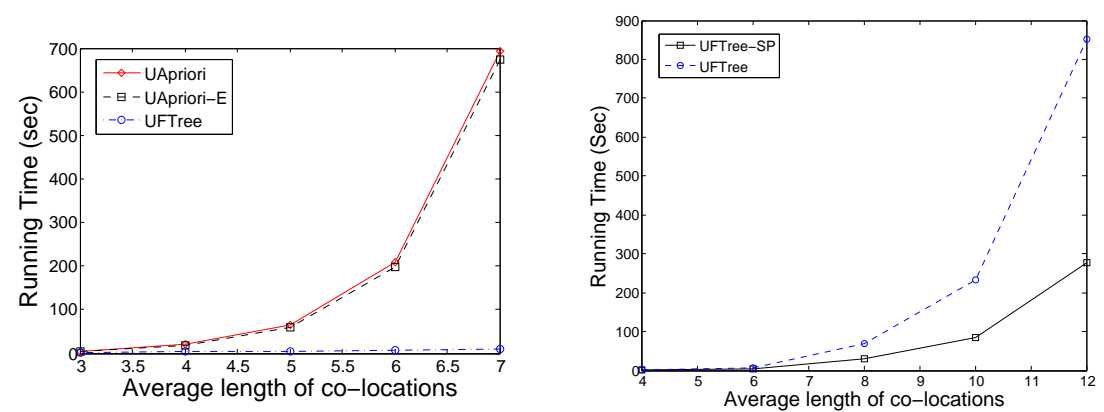

(e) $N_{L}=20 k, \bar{L}: 3 \sim 7, \bar{E}=3, \bar{I}=2$

(f) $N_{L}=20 k, \bar{L}: 4 \sim 12, \bar{E}=3, \bar{I}=3$

Fig. 6: Experiment results on synthetic data.

with probability as the ratio of the number of trip ending points in the small group over that in $e$. 


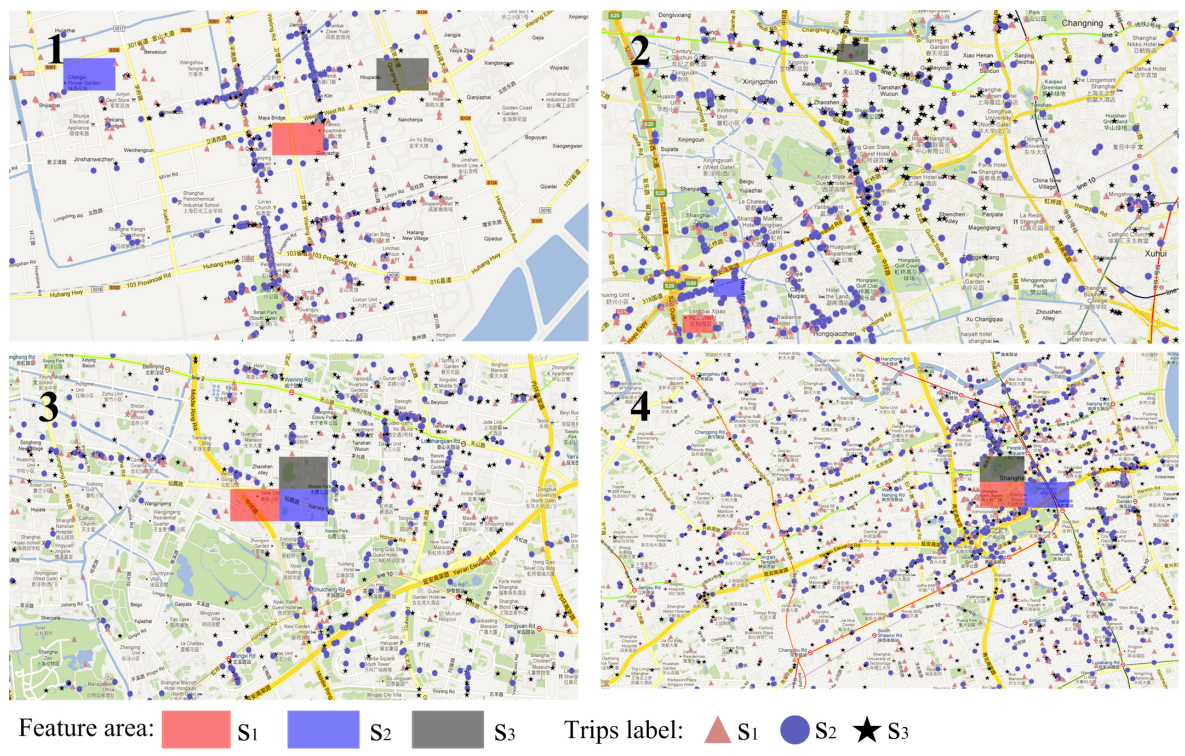

Fig. 7: Four example co-locations from top 10 co-locations on the taxi trip dataset. Rectangle areas represent the spatial features. Their trip ends are represented by a triangle, circle and pentagram respectively.

We ran the mining algorithms on the taxi trips dataset with instance distance threshold as 100 meters. Figure 7 shows 4 example results from the top 10 colocations. Table 2 describes the feature areas of the co-locations in figure 7 and gives the possible reasons for the co-locations. Feature areas in example 1 and 2 have the same characteristics (residences, school zones). Example 3 and 4 show three adjacent features. Especially in example 4, People's Square and People's Park are famous tourism attractions in Shanghai. People often visit them together.

\section{Related Work}

Co-location patterns $[10,15,11]$ and efficient algorithms have been studied by various researchers. An initial summary of results on general spatial co-location mining was proposed in [12]. The authors proposed the notion of user-specified neighborhoods in place of transactions to specify group of spatial items. By doing so, they can adopt traditional association rule mining algorithms, i.e., Apriori [2] to find spatial co-location rules. An extended version of their approaches was presented in [10]. Fast mining algorithms are proposed in [15]. The algorithms combine the discovery of spatial neighborhoods with the mining process. The algorithms work on a given pattern, star, clique, or generic, and calculate the participation index using an extension of a spatial join algorithm. Others have considered complex co-location patterns including negative co-locations [11] and zonal co-locations [6] with dynamic parameters, i.e., repeated specification of 
Table 2: Examples of real data co-location

Co-location Samples with Top Rank Participation Index

1. \{Chengxi Flower Garden \}, \{ Shanxin Apartment $\}$, \{Zongtongwan Garden\}

Explanation: All of those three areas are large and up-scale neighborhoods with many community facilities and amenities.

2. \{Shanghai Longbai Middle School, Longbai Rainforest Preschool\}, \{Longbai No.1 Elementary School, Xijiao Apartment\}, \{Shanghai Tianshan Middle School\}

Explanation: These three areas largely overlap with a school zone.

3. \{Hongxian Unit, Xinle Unit $\}$, \{Hongxian Unit $\},\{$ Xianxia Villa $\}$

Explanation: Three adjacent residential areas.

4. $\{$ People's Square, Shanghai Grand Theatre $\},\{$ People's Square $\}$, \{People's Park\}

Explanation: Three famous adjacent entertainment and tourism areas in Shanghai.

zone and interest measure values according to user preferences. The problem of mining co-location patterns with rare spatial features has been studied in [9]. The authors applied a new measure which considers the maximum participation ratio of the co-location patterns.

In [1], the authors study the problem of frequent pattern mining with uncertain data. They extend the Apriori-based, hyper-structure based, and pattern growth approaches and conclude that experimental behaviour of different classes of algorithms is very different in the uncertain case as compared to the deterministic case. In [13], the authors studied on the probabilistic spatially co-locations and proposed a dynamic programming algorithm which is suitable for parallel computation. They proposed the uncertainty model by introducing the concept of existential probability of an instance. The probability of a possible world can be calculated as the product of the probabilities of presence or absence of all uncertainty instances. By multiplying the probability of possible worlds and the participation index under certain case together, they can get the finial participation index. This model is only considers the probability of existence while our model considers the possible locations of all instances.

The lexicographic tree (enumeration tree) has been used in mining maximal or long pattern association rules. The counting [14] and pruning [5, 16, 8] methods in the search tree to generate association rules are not applicable to our problem. In [14], the authors presented the maximal clique generation method. They modified the Bierstone's algorithm [7] and used the concept of $\alpha$-related to weaken the clique generation process to avoid the edge density is too high.

\section{Conclusion}

In this paper, we formulated the framework for mining co-locations in uncertain database and defined the notion of participation index in the uncertain case. 
We presented the UApriori algorithm to mining co-locations in uncertain data and proposed event level pruning (UApriori-E) to make it more efficient. We presented the uncertain feature tree co-location algorithms and introduced different searching and pruning methods. Our experiment showed that in mining large size co-locations or dealing with datasets with high level of uncertainty, the feature tree algorithm with follow set pruning provided the best performance. In future, we will generalize our model to account for distributions of instances instead of the possible instance model used in this paper.

\section{References}

1. Charu C. Aggarwal, Yan Li, Jianyong Wang, and Jing Wang. Frequent pattern mining with uncertain data. In Proceedings of the 15th ACM SIGKDD, 2009.

2. Rakesh Agrawal and Ramakrishnan Srikant. Fast algorithms for mining association rules in large databases. In Proc. VLDB, 1994.

3. Thomas Bernecker, Tobias Emrich, Hans-Peter Kriegel, Matthias Renz, Stefan Zankl, and Andreas Züfle. Efficient probabilistic reverse nearest neighbor query processing on uncertain data. Proc. VLDB Endow., 2011.

4. Coen Bron and Joep Kerbosch. Algorithm 457: finding all cliques of an undirected graph. Commun. ACM, 1973.

5. D. Burdick, M. Calimlim, J. Flannick, J. Gehrke, and T. Yiu. Mafia: a maximal frequent itemset algorithm. TKDE, 2005.

6. Mete Celik, James M. Kang, and Shashi Shekhar. Zonal co-location pattern discovery with dynamic parameters. In Proceedings of the Seventh IEEE ICDM, 2007.

7. D.G. Corneil G.D. Mulligan. Corrections to bierstone's algorithm for generating cliques. Commun. ACM, 1972.

8. K. Gouda and M.J. Zaki. Efficiently mining maximal frequent itemsets. In ICDM, 2001.

9. Yan Huang, Jian Pei, and Hui Xiong. Mining co-location patterns with rare events from spatial data sets. Geoinformatica, 2006.

10. Yan Huang, Shashi Shekhar, and Hui Xiong. Discovering colocation patterns from spatial data sets: A general approach. TKDE, 2004.

11. Robert Munro, Sanjay Chawla, and Pei Sun. Complex spatial relationships. In Proceedings of the Third IEEE ICDM, 2003.

12. Shashi Shekhar and Yan Huang. Discovering spatial co-location patterns: A summary of results. In SSTD, 2001.

13. Lizhen Wang, Pinping Wu, and Hongmei Chen. Finding probabilistic prevalent colocations in spatially uncertain data sets. IEEE TKDE, 2013.

14. M.J. Zaki. Scalable algorithms for association mining. TKDE, 2000.

15. Xin Zhang, Nikos Mamoulis, David W. Cheung, and Yutao Shou. Fast mining of spatial collocations. In ACM SIGKDD, 2004.

16. Qinghua Zou, W.W. Chu, and Baojing Lu. Smartminer: a depth first algorithm guided by tail information for mining maximal frequent itemsets. In ICDM, 2002. 